ఠ

Open Access Full Text Article

REVIEW

\title{
Anti-N-methyl-D-aspartate receptor encephalitis mimics neuroleptic malignant syndrome: case report and literature review
}

This article was published in the following Dove Medical Press journal:

Neuropsychiatric Disease and Treatment

\author{
Hai-Yang Wang' \\ Tian $\mathrm{Li}^{2,3}$ \\ Xue-Lin $\mathrm{Li}^{4}$ \\ Xiao-Xia Zhang' \\ Zhong-Rui Yan' \\ Yang $\mathrm{Xu}{ }^{\prime}$
}

'Department of Neurology, Jining No I People's Hospital, Jining, Shandong Province, People's Republic of China; ${ }^{2}$ Department of Cardiology, Jining No I People's Hospital, Jining, Shandong Province, People's Republic of China; ${ }^{3}$ Department of Internal Medicine-Cardiovascular, Qilu Hospital of Shandong University, Jinan, Shandong Province, People's Republic of China; ${ }^{4}$ Department of Intensive Care Unit, Jining No I People's Hospital, Jining, Shandong Province, People's Republic of China
Correspondence: Yang Xu Department of Neurology, Jining No I People's Hospital, No 6, Jiankang Road, Jining, Shandong Province 2720I I,

People's Republic of China

Tel +86 I 3963750808

Email jnkfxy@।63.com
Background: Anti-N-methyl-D-aspartate (anti-NMDA) receptor encephalitis is a severe autoimmune disease characterized by complicated psychiatric and neurological symptoms and a difficult diagnosis. This disorder is commonly misdiagnosed, and diagnosis is often delayed. The clinical signs can mimic other psychiatric abnormalities, such as neuroleptic malignant syndrome (NMS) that is usually caused by antipsychotic exposure. This fact raises the question of whether the symptoms common to NMS are due to anti-NMDA receptor encephalitis or established NMS.

Cases presentation: We describe a rare case of a 29-year-old male without psychiatric history who initially presented with a fever, altered consciousness, behavioral changes, rigidity, and elevated creatine kinase. He was initially diagnosed with NMS. NMS-like symptoms did not improve with active treatments and disappeared for a long period after discontinuing antipsychotics. The patient gradually developed a complicated disease progression, including speech impairment, mutism, and movement disorders, and symptom progression led to the final diagnosis of anti-NMDA receptor encephalitis. The related pathophysiological mechanisms, clinical features, and treatment of this disease are reviewed.

Conclusion: We highlight that the natural progress of anti-NMDA receptor encephalitis can mimic the symptoms of NMS and NMS-like features could be due to anti-NMDA receptor encephalitis upon antipsychotic exposure, and not true NMS. Clinically, the suspicion of NMS may serve as a significant alarm to suspect anti-NMDA receptor encephalitis and lead neurologists or psychiatrists to investigate such a diagnosis.

Keywords: acute psychosis, anti-NMDA receptor encephalitis, autoimmune encephalitis, neuroleptic malignant syndrome, antipsychotics, diagnosis

\section{Introduction}

Anti-N-methyl-D-aspartate (NMDA) receptor encephalitis, a severe form of autoimmune encephalitis, is characterized by a wide spectrum of neuropsychiatric symptoms and is associated with autoantibodies against the NMDA receptor. ${ }^{1}$ In the early phases of the disease, symptoms are often nonspecific (ie, headache, fever, anxiety, and tiredness), which are usually followed by a psychotic stage (ie, delusions, hallucinations, and bizarre behavior). ${ }^{2}$ Cognitive dysfunction, speech dysfunction (pressured speech, verbal reduction, and mutism), and movement disorders are noted in patients with anti-NMDA receptor encephalitis. ${ }^{3}$ Over time, decreased breathing, increased blood pressure, and a faster heart rate typically develop. ${ }^{3}$

This disorder is commonly misdiagnosed, and diagnosis is often delayed. Auxiliary examinations are frequently normal, and clinical signs can mimic other psychiatric 
abnormalities, such as neuroleptic malignant syndrome (NMS) that is usually caused by antipsychotic exposure. Timely and proper treatment can significantly improve the clinical outcome of patients, but prompt intervention requires early recognition. Herein, we present a rare case of antiNMDA receptor encephalitis in a patient who was initially diagnosed with NMS; we further highlight the NMS-like clinical features that led to this diagnosis. Antipsychotic medications are likely to be prescribed for primary psychiatric symptoms, an action that has raised an unresolved question as to whether NMS-like clinical features are due to anti-NMDA receptor encephalitis or to an established NMS.

\section{Case presentation}

A 29-year-old male with no history of psychiatric illness presented to a district general hospital with a 2-week history of acute psychiatric symptoms, memory deficit, a reduced level of consciousness, new behavioral changes including wandering and babbling, and seizures in the context of a new fever $\left(38.9^{\circ} \mathrm{C}\right)$. A computed tomography (CT) scan of the brain and lungs and analysis of cerebrospinal fluid (CSF) did not reveal any abnormalities. The patient was hospitalized for several days and treated with levetiracetam and valproate sodium. Acyclovir was also started empirically to treat a possible Herpes simplex virus (HSV) infection. His fever continued with temperature between $37.5^{\circ} \mathrm{C}$ and $39.6^{\circ} \mathrm{C}$. Blood pressure elevation was initially noted. His blood pressure peaked at 170/95 $\mathrm{mmHg}$ (baseline was $125 / 70 \mathrm{mmHg}$ ), and systolic pressure fluctuation $\geq 25 \mathrm{mmHg}$ occurred within 24 hours. The patient's mental status progressively worsened during treatment; he started having episodes of confusion, shouting, and extreme fear. The patient experienced both visual and auditory hallucinations, eg, auditory hallucinations of voices, talking, and laughing to himself. After 6 days of treatment, the patient's family requested that he be discharged from the hospital due to a lack of improvement in his condition.

The patient presented to the authors for further assessment. Three weeks after onset of initial symptoms, he was diagnosed with acute psychosis and possible viral encephalitis in our hospital and started on olanzapine (10 mg daily) for his acute psychiatric symptoms and foscarnet sodium empirically to treat a possible HSV infection. Despite the treatment with olanzapine, his mental status did not improve; he continued to show symptoms of a psychiatric disorder with manic-like episodes and aggression toward the doctors and nurses. Gradually, the patient displayed speech impairment and a marked rigidity of the neck and torso.
The patient was re-evaluated by a neurologist and psychiatrist. A physical examination revealed that he had a fever $\left(39.5^{\circ} \mathrm{C}\right)$, a heart rate of 112 beats $/ \mathrm{min}$, high blood pressure $(160 / 90 \mathrm{mmHg})$, and profuse sweating. A second CT brain scan, an electrocardiogram, and a 24-hour ambulatory electroencephalography were all normal. CSF results revealed 10 cells $/ \mathrm{mm}^{3}$, a protein level of $0.34 \mathrm{~g} / \mathrm{L}$, and a sugar level of $2.4 \mathrm{mmol} / \mathrm{L}$. The infectious index of the CSF, such as for bacteria, fungus, acid-fast bacillus, Cryptococcus, TORCH-IgM/IgG, quantification of immunoglobulin, and Japanese encephalitis, was negative, as were evaluations for HIV, cytomegalovirus, influenza, Borrelia burgdorferi, and syphilis. Blood work showed a creatine kinase (CK) value of 1,096 U/L, a normal C-reactive protein level, and a marginally elevated white cell count of $13.87 \times 10^{9} / \mathrm{L}$. Based on the presence of mental status changes, hyperthermia, muscular rigidity, high $\mathrm{CK}$ value (four times higher than the normal level), and autonomic dysfunction after recent exposure to olanzapine, ${ }^{4,5}$ olanzapine was discontinued and he was diagnosed with NMS. He was then treated with midazolam $(0.5 \mathrm{mg} / \mathrm{kg}$ per hour, intravenous), a benzodiazepine.

The patient received supportive care and intravenous benzodiazepines, but even after the CK level returned to normal, he did not exhibit any improvement. During medical treatment, the patient developed mutism and cycling-like movements of his legs. At this stage, we reviewed the entire disease course. Even though his symptoms met many features of NMS, we did not think it was NMS since there were symptoms prior to antipsychotic administration, eg, hyperthermia and some acute psychiatric symptoms, including mood swings, anxiety, and behavioral changes. We found no evidence that separate or combined use of levetiracetam, valproate sodium and acyclovir causes NMS.

Further investigations were performed since the disease progression was unexpected. Immunological analyses were positive for specific anti-NMDA receptor antibodies in both CSF (IgG, 1:32) and serum (IgG, 1:320) (Figure 1A and B). As such, the anti-NMDA receptor encephalitis diagnosis was confirmed on the 12th day after being admitted to our hospital. Various examinations, including antibodies for paraneoplastic syndromes, tumor markers, abdominal and pelvic CT scans, and ultrasounds of the reproductive system, were performed to exclude neoplasm associated with anti-NMDA receptor encephalitis; each of these results was negative.

The patient was treated with a combination regimen of plasmapheresis and methylprednisolone. The patient gradually improved and was discharged to the care of his family after 5 weeks. Significantly, some NMS-like symptoms 

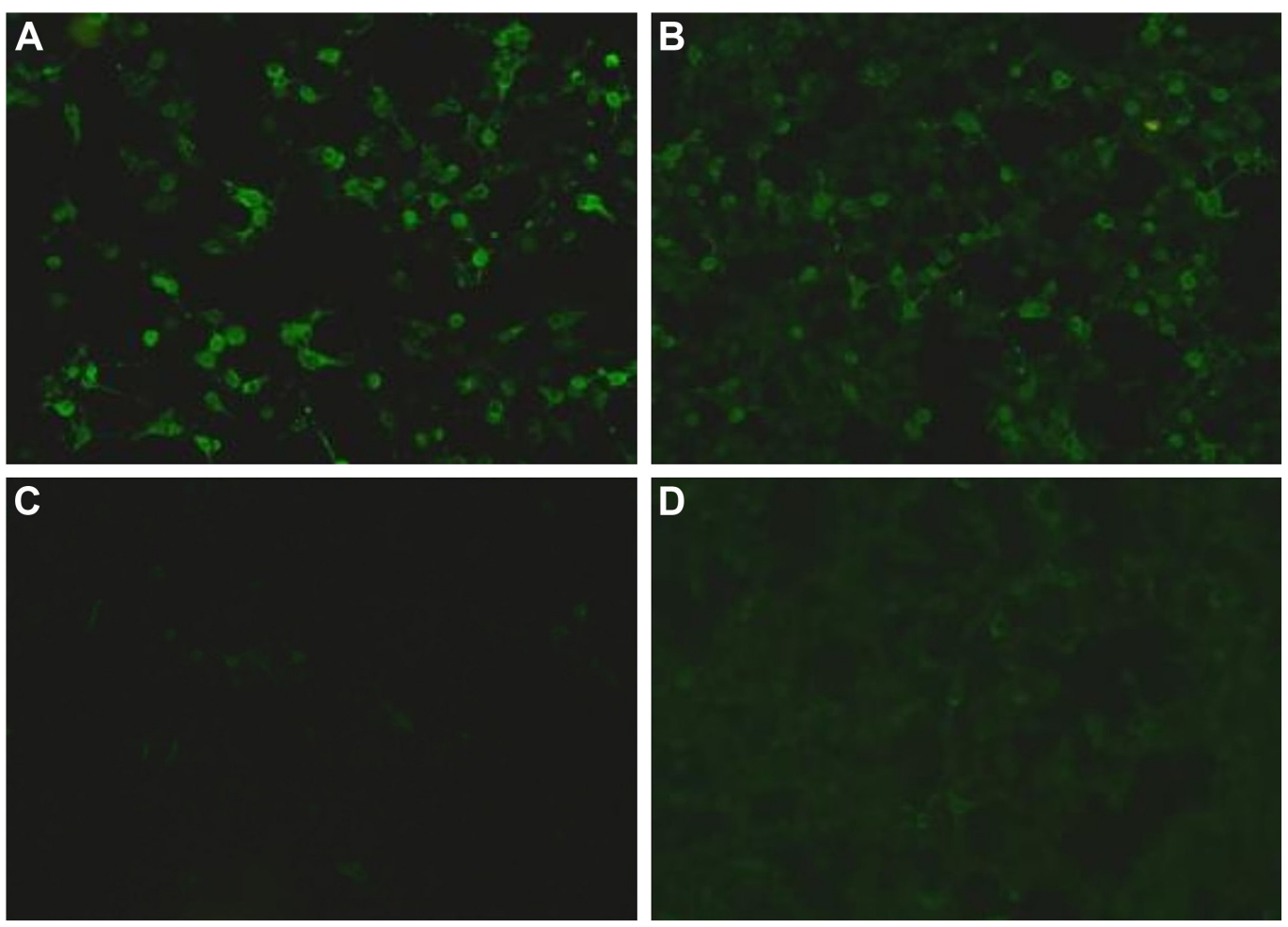

Figure I Anti-NMDA receptor antibodies in patient's cerebrospinal fluid and serum. Anti-NMDA receptor antibodies were positive in cerebrospinal fluid (IgG, I:32) (A) and serum (lgG, I:320) (B) before immunotherapy. The level of anti-NMDA receptor antibodies significantly decreased in cerebrospinal fluid (IgG, I:3.2) (C) and serum ( $\operatorname{lgG}, \mathrm{I}: 32$ ) (D) after 5 months of immunotherapy.

Abbreviation: NMDA, N-methyl-D-aspartate.

(such as cloudy consciousness, hypermyotonia, and autonomic dysfunction) did not disappear completely 1 month after discontinuing olanzapine. His symptoms continued to improve after discharge, and the level of antibodies significantly decreased in cerebrospinal fluid (IgG, 1:3.2) and serum (IgG, 1:32) (Figure 1C and D) after 5 months of immunotherapy. These NMS-like symptoms diminished and ultimately disappeared within months.

\section{Discussion}

Diagnosing anti-NMDA receptor encephalitis can be challenging because its clinical features can overlap with other conditions, especially psychiatric illnesses, ${ }^{6}$ and ancillary investigations are frequently negative. ${ }^{3}$ While there is growing literature on the differential diagnosis of anti-NMDA receptor encephalitis with psychiatric disorders, it is rarely described that anti-NMDA receptor encephalitis could mimic NMS.

Anti-NMDA receptor encephalitis has significant clinical overlap with NMS (Table 1). NMS is a rare but potentially dangerous and rapidly progressive condition, with considerable morbidity and mortality risks. ${ }^{7}$ Muscular rigidity, hyperthermia, altered consciousness, CK elevation, and autonomic dysfunction are the required diagnostic criteria for NMS. ${ }^{4,5,7}$ These clinical symptoms are frequently present in cases of anti-NMDA receptor encephalitis, with one exception, that is, elevated CK. Therefore, it is not surprising that the patient was initially diagnosed with NMS. He exhibited an altered mental status, muscle rigidity, leukocytosis, elevated CK, a rapid heart rate, high blood pressure, dysautonomia, and was also exposed to antipsychotics. However, although these features did fit some of the NMS characteristics, this diagnosis did not explain all symptoms, nor did it explain the disease progression. The other symptoms led us to re-review the disease onset and course in our patient. He was eventually diagnosed with anti-NMDA receptor encephalitis: he had abnormal (psychiatric) behavior, cognitive dysfunction, speech dysfunction, seizures, movement disorder, autoantibodies against NMDA receptors, and NMS-like clinical features.

It is worth noting that NMS may be caused by recent antipsychotic exposure or it may possibly be a natural progression of anti-NMDA receptor encephalitis itself in a subset of patients. Approximately, three-quarters of patients are first assessed by a psychiatrist for their primary psychiatric symptoms, ${ }^{6}$ and patients may be more likely to receive 
Table I Clinical similarities, differences, and diagnostic features in anti-NMDA receptor encephalitis and NMS

\begin{tabular}{|c|c|c|}
\hline & Anti-NMDA receptor encephalitis & NMS \\
\hline \multirow{7}{*}{$\begin{array}{l}\text { Diagnostic criteria/ } \\
\text { features }\end{array}$} & - Mental status alteration & - Mental status alteration \\
\hline & - Hyperthermia & - Hyperthermia \\
\hline & - Rigidity/abnormal postures & - Rigidity \\
\hline & - Psychiatric behavior & \\
\hline & - Autonomic dysfunction & - Autonomic dysfunction \\
\hline & & - Elevation of CK by at least four times the upper limit of normal \\
\hline & - Seizures & \\
\hline Prodromal symptoms & Fever or headache & Changes in mental status \\
\hline Onset & Rapid onset (less than 3 months) & Within 24 hours ( $16 \%)$, within I week (66\%), within 30 days (96\%) \\
\hline \multirow[t]{5}{*}{ Other reported features } & Movement disorder & Tremor, sialorrhea, akinesia, dystonia, trismus, and myoclonus \\
\hline & Speech dysfunction & Dysarthria and dysphagia \\
\hline & Central hypoventilation & Leukocytosis \\
\hline & Cognitive dysfunction & Metabolic acidosis \\
\hline & & Elevated catecholamines electrolyte changes \\
\hline CK & No related reports & By at least four times the upper limit of normal \\
\hline CSF & Pleocytosis or oligoclonal bands & Generally normal \\
\hline Neuroimaging studies & Generally normal & Generally normal \\
\hline EEG & $\begin{array}{l}\text { Focal or diffuse, slow or disorganized activity, } \\
\text { epileptic activity, extreme delta brush or nomal }\end{array}$ & Nonfocal generalized slowing (54\%) \\
\hline Outcome & $\begin{array}{l}\text { About } 80 \% \text { patients recovered well } \\
\text { (mRS score } 0-2 \text { ) }\end{array}$ & $\begin{array}{l}\text { Mean recovery time is } 7-10 \text { days after drug discontinuation, and } \\
\text { virtually all patients recover within } 30 \text { days }\end{array}$ \\
\hline
\end{tabular}

Abbreviations: NMDA, N-methyl-D-aspartate; NMS, neuroleptic malignant syndrome; CK, creatine kinase; CSF, cerebrospinal fluid; EEG, electroencephalography; mRS, modified Rankin Scale.

antipsychotics at this stage. Although our patient received olanzapine, which is known to cause NMS, his symptoms of hyperthermia and reduced level of consciousness appeared early, that is, before treatment with this drug. Furthermore, discontinuation of olanzapine and the use of benzodiazepine is an effective therapy for NMS, ${ }^{5,7}$ but it did not significantly improve his condition until we commenced immunemodulating treatment. Once recognized and antipsychotics are stopped, NMS is usually self-limited. The mean recovery time is $7-10$ days after drug withdrawal, and virtually all patients recover within 30 days. ${ }^{5}$ However, symptoms of mental status changes, muscular rigidity, and autonomic dysfunction in our patients did not disappear completely 1 month after discontinuing olanzapine. Our case suggests that the symptoms common to NMS could be due to antiNMDA receptor encephalitis in patients with antipsychotic exposure and not due to true NMS.

The NMS-like features of anti-NMDA receptor encephalitis are not unique and can be found in many other cases of encephalitis and brain infection and/or inflammation. ${ }^{8}$ There have been several case reports of other immunemediated encephalitides that present with NMS-like features, including progressive encephalomyelitis with rigidity and myoclonus $^{9}$ and anti-voltage-gated potassium channel complex encephalitis. ${ }^{10}$ Additionally, benzodiazepines are the first line of clinical intervention for $\mathrm{NMS}^{5}$ and are also used for the auxiliary treatment of the psychotic anti-NMDA receptor encephalitis psychotic symptoms, ${ }^{11}$ which may have similar pathophysiological properties. Therefore, our case suggests that the natural course of this disease could mimic the features of NMS and indeed may describe a common disease progression.

In patients treated with antipsychotic drugs, it may be unclear whether the symptoms common to NMS are due to anti-NMDA receptor encephalitis or to an established NMS. Some studies suggest that NMS might be considered a differential diagnosis for anti-NMDA receptor encephalitis. ${ }^{12,13}$ While these studies highlight that some anti-NMDA receptor encephalitis symptoms could overlap with NMS clinical features, they did not discuss deeply whether NMS is caused by antipsychotics or if NMS is a feature of the anti-NMDA receptor encephalitis itself. In one retrospective study,,$^{14}$ for all suspected NMS patients, the main diagnostic criterion was that antipsychotic drugs were administered hours or days before the emergent high temperature. This study acknowledged that they did not have enough information on these 
patients to strictly confirm the NMS diagnosis. However, our case highlights that the natural process of anti-NMDA receptor encephalitis could mimic NMS symptoms, and the symptoms common to NMS may only emerge in an anti-NMDA receptor encephalitis patient due to antipsychotic exposure, a phenomenon that is different from other studies. ${ }^{15,16}$

An important alternative understanding is the likelihood that these diagnoses are not mutually exclusive. In fact, patients with anti-NMDA receptor encephalitis are at high risk for NMS if treated with antipsychotics. Therefore, some patients could have encephalitis which could worsen, and they can subsequently develop NMS after receiving antipsychotics. In other words, they could have both conditions, although their prognosis would be determined by the encephalitis which may persist after NMS resolves. ${ }^{17-19}$

Although the natural course of anti-NMDA receptor encephalitis mimics the symptoms of NMS, it may affect the basal ganglia, hypothalamus, and brainstem regulatory systems through different pathophysiological mechanisms. There is a convincing clinical evidence that $\mathrm{D}_{2}$ dopamine receptor blockade may play a significant role in NMS pathophysiology. ${ }^{5}$ Central dopaminergic systems are associated with temperature regulation, muscular tension, and movement. ${ }^{7}$ Muscular rigidity is caused by $\mathrm{D}_{2}$ dopamine receptor blockade, and this action leads to basal ganglia dysfunction, as observed in Parkinson's disease. ${ }^{7}$ Dysregulation of hypothalamic dopamine receptor blockade leads to hyperthermia in NMS. ${ }^{7}$ Changes in dopamine neurotransmission in the reticular activating system in the brainstem may be involved with altered consciousness. ${ }^{7}$ Antipsychotic-induced dopamine blockade in the basal ganglia, hypothalamus, and brainstem regulatory systems cause NMS symptoms. However, decreased NMDA receptors could also affect dopaminergic systems. ${ }^{1}$ NMDA receptors can control the release of dopamine through direct presynaptic action on dopaminergic terminals. ${ }^{20}$ Therefore, NMDA receptor antibodies could play a role in dopamine blockade, which may lead to NMS-like symptoms in patients without antipsychotic exposure.

The overlap of anti-NMDA receptor encephalitis and NMS confounds diagnosis and delays treatment, both of which are challenging. Clinically, many patients are likely to receive antipsychotic medications. ${ }^{6}$ Given the risks of NMS and the difficulty in distinguishing NMS from the early natural progression of anti-NMDA receptor encephalitis, antipsychotics should be used with caution to treat the symptoms of psychosis associated with anti-NMDA receptor encephalitis. Psychiatric symptoms gradually improve after immunotherapy, despite many antipsychotic medications that show potential benefits. A recent study suggests that anti-NMDA receptor encephalitis may have an increased susceptibility to antipsychotics and doctors should use extreme caution in the use of antipsychotic drugs in patients. Our case highlights that the symptoms common to NMS could be due to anti-NMDA receptor encephalitis in patients exposed to antipsychotics. However, this phenomenon may occur in only a proportion of patients. Therefore, it is important to consider and rule out anti-NMDA receptor encephalitis when treating patients with psychosis of unclear etiology to reduce the risk of unnecessary and unhelpful antipsychotic drug exposure.

\section{Conclusion}

This case highlights that the natural progression of antiNMDA receptor encephalitis could mimic the symptoms of NMS, and NMS-like features could be due to anti-NMDA receptor encephalitis in patients exposed to antipsychotics and not necessarily due to true NMS; this example is rare in the literature. Anti-NMDA receptor encephalitis could not cause NMS, but some NMS-like clinical features could be observed in the course of anti-NMDA receptor encephalitis with or without antipsychotics. Furthermore, these syndromes are overlapping in symptoms and diagnostic criteria, and hence further investigations should deeply explore the underlying brain pathology between anti-NMDA receptor and NMS. Suspicion of NMS may be a significant alarm to suspect anti-NMDA receptor encephalitis and lead neurologists and/or psychiatrists to investigate such a diagnosis.

\section{Ethics statement}

Legal guardian of the patient has provided written informed consent for the case details to be published. The present study was approved by the Ethics Committee of the Jining No 1 People's Hospital. All subjects gave written informed consent to participate in the study in accordance with the Declaration of Helsinki.

\section{Acknowledgments}

We appreciate Professor Bingwei Zhang (Department of Neurology and Psychiatry, First Affiliated Hospital of Dalian Medical University) very much for his constructive suggestions on our manuscript.

\section{Author contributions}

HYW wrote the manuscript. HYW, TL, XLL, and YX actively participated in the data acquisition and prepared the table. HYW, XXZ, ZRY, and YX reviewed the literature and 
participated in data interpretation. All authors contributed to the final manuscript review and editing. All authors contributed to data analysis, drafting or revising the article, gave final approval of the version to be published, and agree to be accountable for all aspects of the work.

\section{Disclosure}

The authors report no conflicts of interest in this work.

\section{References}

1. Dalmau J, Lancaster E, Martinez-Hernandez E, Rosenfeld MR, BaliceGordon R. Clinical experience and laboratory investigations in patients with anti-NMDAR encephalitis. Lancet Neurol. 2011;10(1):63-74. doi:10.1016/S1474-4422(10)70253-2

2. Kayser MS, Dalmau J. Anti-NMDA receptor encephalitis, autoimmunity, and psychosis. Schizophr Res. 2016;176(1):36-40. doi:10.1016/j. schres.2014.10.007

3. Graus F, Titulaer MJ, Balu R, et al. A clinical approach to diagnosis of autoimmune encephalitis. Lancet Neurol. 2016;15(4):391-404. doi:10.1016/S1474-4422(15)00401-9

4. American Psychiatric Association. DSM-IV-TR: Diagnostic and Statistical Manual of Mental Disorders, Text Revision. Washington, DC: American Psychiatric Association; 2000.

5. Ware MR, Feller DB, Hall KL. Neuroleptic malignant syndrome: diagnosis and management. Prim Care Companion CNS Disord. 2018; 20(1):17r02185. doi:10.4088/PCC.17r02185

6. Gurrera R. F186. Common non-behavioral clinical features of antiNMDAR encephalitis presenting as a psychiatric disorder. Biol Psychiatry. 2018;83(9):S311. doi:10.1016/j.biopsych.2018.02.800

7. Velamoor R. Neuroleptic malignant syndrome: a neuro-psychiatric emergency: recognition, prevention, and management. Asian J Psychiatr. 2017;29:106-109. doi:10.1016/j.ajp.2017.05.004

8. Caroff SN, Mann SC, Gliatto MF, Sullivan KA, Campbell EC. Psychiatric manifestations of acute viral encephalitis. Psychiatr Ann. 2001; 31(3):193-204. doi:10.3928/0048-5713-20010301-10

9. Xu Z, Prasad K, Yeo T. Progressive encephalomyelitis with rigidity and myoclonus in an intellectually disabled patient mimicking neuroleptic malignant syndrome. J Mov Disord. 2017;10(2):99-101. doi:10.14802/ jmd. 16058
10. Iyer A, Mctague A, Curran A, Inbasagaran A, Vincent A, Kneen R. VGKC-complex antibody mediated encephalitis presenting with psychiatric features and neuroleptic malignant syndrome - further expanding the phenotype. Dev Med Child Neurol. 2012;54(6):575-576. doi:10.1111/j. 1469-8749.2012.04249.x

11. Kayser MS, Dalmau J. Anti-NMDA receptor encephalitis in psychiatry. Curr Psychiatry Rev. 2011;7(3):189-193. doi:10.2174/1573400 11797183184

12. González-Valcárcel J, Rosenfeld MR, Dalmau J. Differential diagnosis of encephalitis due to anti-NMDA receptor antibodies. Neurologia. 2010;25(7):409-413.

13. Guasp M, Dalmau J. Encephalitis associated with antibodies against the NMDA receptor. Med Clin (Barc). 2018;151(2):71-79. doi:10.1016/j. medcli.2017.10.015

14. Lejuste F, Thomas L, Picard G, et al. Neuroleptic intolerance in patients with anti-NMDAR encephalitis. Neurol Neuroimmunol Neuroinflamm. 2016;3(5):e280. doi:10.1212/NXI.0000000000000280

15. Rozier M, Morita D, King M. Anti-N-methyl-D-aspartate receptor encephalitis: a potential mimic of neuroleptic malignant syndrome. Pediatr Neurol. 2016;63:71-72. doi:10.1016/j.pediatrneurol.2016. 03.023

16. Kiani R, Lawden M, Eames P, et al. Anti-NMDA-receptor encephalitis presenting with catatonia and neuroleptic malignant syndrome in patients with intellectual disability and autism. BJPsych Bull. 2015;39(1):32-35. doi:10.1192/pb.bp.112.041954

17. Caroff SN, Mann SC, Mccarthy M, Naser J, Rynn M, Morrison M. Acute infectious encephalitis complicated by neuroleptic malignant syndrome. J Clin Psychopharmacol. 1998;18(4):349-351.

18. Caroff SN, Campbell EC. Risk of neuroleptic malignant syndrome in patients with NMDAR encephalitis. Neurol Sci. 2015;36(3):479-480. doi:10.1007/s10072-014-2022-z

19. Caroff SN, Mann SC, Campbell EC. Anti-N-methyl-D-aspartate receptor encephalitis and risk of neuroleptic malignant syndrome. Pediatr Neurol. 2017;66:e3. doi:10.1016/j.pediatrneurol.2016.10.005

20. Salamone A, Zappettini S, Grilli M, et al. Prolonged nicotine exposure down-regulates presynaptic NMDA receptors in dopaminergic terminals of the rat nucleus accumbens. Neuropharmacology. 2014;79(4): 488-497. doi:10.1016/j.neuropharm.2013.12.014
Neuropsychiatric Disease and Treatment

\section{Publish your work in this journal}

Neuropsychiatric Disease and Treatment is an international, peerreviewed journal of clinical therapeutics and pharmacology focusing on concise rapid reporting of clinical or pre-clinical studies on a range of neuropsychiatric and neurological disorders. This journal is indexed on PubMed Central, the 'PsycINFO' database and CAS,

\section{Dovepress}

and is the official journal of The International Neuropsychiatric Association (INA). The manuscript management system is completely online and includes a very quick and fair peer-review system, which is all easy to use. Visit http://www.dovepress.com/testimonials.php to read real quotes from published authors. 